\title{
Formation of Nations in a Welfare-State MiNDED WORLD
}

\author{
NIR DAGAN \\ Economics Department, Brown University \\ OSCAR VOLIJ \\ Economics Department, Brown University \\ and \\ Hebrew University, Jerusalem
}

\begin{abstract}
We model the endogenous formation of nations in a world economy where nations apply redistributive policies. We show that people's preference for stronger redistributive policies may lead to greater inequality in the world's distribution of income as a result of rich individuals tending to form their own nations. By the same token, stable economic integration occurs only when redistributive policies are not too strong.
\end{abstract}

\section{Introduction}

One of the main purposes of taxation is the redistribution of income, and one of the rationales for redistribution is that it brings about a better society in terms of income equality. Other things being equal (namely, disregarding other purposes and effects of taxation, such as providing public goods and incentives), a nonregressive income tax improves the distribution of income in society. On the other hand, it is not clear how far a society is willing to go in accepting a strong redistributive policy.

Most treatments of taxation consider a given society with a benevolent government that tries to maximize a social welfare function; a few papers

Nir Dagan, Economics Department, Brown University, Providence, RI 02912 (nir@nirdagan. com). Oscar Volij, Economics Department, Brown University, Providence, RI 02912 and Hebrew University, Jerusalem 91905, Israel (Oscar_Volij@Brown.edu).

We acknowledge the hospitality of the Economics Department at Bar-Ilan University, where this paper was highly improved. We also thank our European hosts, CentER and Universidad Carlos III, where the basic ideas were conceived. Finally, we appreciate the comments of two referees, who encouraged us to strengthen our results and to explore the issue of capital mobility.

Received 17 November 1998; Accepted 15 June 1999.

(C) 2000 Blackwell Publishers, Inc.

Journal of Public Economic Theory, 2 (2), 2000, pp. 157-181. 
apply game-theoretic models in which the government is responsive to pressures exerted by the voters. An alternative game-theoretic approach, whereby the formation of jurisdictions within society is endogenously determined, was introduced by Wooders (1978), Guesnerie and Oddou (1981), and Greenberg and Weber (1986). There, however, the issue is the provision of a public good. In the present paper we adopt a similar approach, but apply it to the analysis of income redistribution. Our primary purpose is to investigate the effects of redistributive policies on the distribution of income worldwide. A close predecessor is Bennett and Wooders (1979) where a similar approach is applied to the analysis of the effects of redistributive policies to efficiency. Our solution concept incorporates the possibility of any group of individuals forming a separate nation with its own proportional tax policy. Consequently, our approach takes into consideration the fact that if the tax burden is too heavy, some individuals may prefer to secede and form their own nation.

In order to focus on the redistributive role of taxation we adopt a general equilibrium framework in which taxes are nondistortionary. Though individuals differ in their endowments, they have the same preferences, which are defined over their consumption bundles of private goods and on a measure of income redistribution. We assume no cultural links or historical background to unite people, and no form of atavism separates individuals. Summarizing, we model a world in which all individuals are identical except for their initial wealth.

The individuals' concern about income redistribution is what we call "welfare-state mindedness." In the model, individuals do not want to live in a society where the government does not provide a minimum level of income. Once this minimum level is guaranteed by the government for all individuals in society, agents care only for their own consumption. Therefore, provided the required minimum level is guaranteed, the only reason for an agent to join another nation or to emigrate from his own is an improvement of his own material well-being. This kind of welfare-state mindedness may seem too extreme but was neatly captured in a frontpage New York Times article on December 13, 1996, quoting a representative Norwegian who said: "We are a very social democratic society.... It may be costly, but there is social peace. There are no poor people in Norway and I don't want to see any."

We look for an international equilibrium, which is a stable partition of the set of individuals into nations. By stable we mean that no group of individuals would be better off by forming a new nation. In the equilibrium, not only is the partition of the world into nations endogenously determined, but also each nation's redistributive policy. It can be argued that our equilibrium concept abstracts from political institutions that may be relevant for the determination of the nation's redistributive policy. Our results indicate, however, that the precise modeling of these political institutions is irrelevant, provided people have the ability to form their 
own nations with their own institutions, without the consent of others. Alternatively one can say that the only institutions that will be observed in equilibrium are those consistent with our endogenously determined redistributive policy.

Unlike in models where the main role of taxation is to finance a public good (see, e.g., Westhoff 1977 and Greenberg and Weber 1986), our equilibria are typically constituted by nations of very different people. It is not generally true that individuals with similar incomes cluster together. Unlike most treatments of migration (see, e.g., Myers 1990; Wildasin 1991; Hercowitz and Pines 1991; and Cukierman, Hercowitz, and Pines 1994), where there is free mobility between jurisdictions within a nation, in our model it may well be the case that an individual prefers, in equilibrium, a nation other than his own. This desire to emigrate does not upset the equilibrium because, in order to immigrate, an individuals needs the consent of the absorbing country. Although the assumption of free mobility is certainly appropriate when one deals with jurisdictions within a nation, it is extremely unrealistic when the mobility is across countries, as anyone who has tried to migrate from one country to another can testify.

Another result relates to the issue of economic integration. In order to achieve economic integration, individuals should not be excessively welfare-state minded. It is shown that there is a level of welfare-state mindedness whose corresponding redistributive policy guarantees economic integration, which results not only in efficient production but also yields a world distribution of income that Lorenz dominates any other equilibrium income distribution achieved under a stronger policy. Further, once beyond that critical level, the stronger the distributive policy, the less equal the worldwide distribution of income. The intuition behind this result is that too strong a redistributive policy may lead to a world with many relatively egalitarian societies that nonetheless differ in their per capita incomes, while a weaker policy would yield a world with less egalitarian nations but with similar average income levels. Matters become aggravated when the incomes policy pursued is too vigorous, because the division of the world into very different nations (in terms of their capitallabor ratio) carries an efficiency loss with it.

Our model assumes no capital mobility across nations. This assumption is behind the fact that equilibrium nations may have heterogeneous populations. When there is perfect capital mobility, many of our results are strengthened. In particular, individuals' preference for redistribution have no effect on the final outcome. In fact, with perfect capital mobility no income distribution takes place.

The paper is organized as follows: After presenting the basic definitions related to national economies in Section 2, we discuss the central solution concept of an international equilibrium in Section 3. Section 4 gives a characterization of international equilibria, showing also their existence and essential uniqueness. The relation between government inter- 
vention, income distribution, and integration of the world economy is analyzed in Section 5. Section 6 discusses the related literature. Theorem proofs are in the Appendix.

\section{The Model}

\subsection{National Equilibrium}

Let $T=\{1,2\}$ be a set of two types, with the associated characteristics $\left(K_{i}, L_{i}, U_{i}\right), i=1,2$. Here $K_{i}$ and $L_{i}$ are the initial capital and labor endowments of an individual of type $i$ and $U_{i}: \mathbb{R}_{+}^{2} \rightarrow \mathbb{R}$ is his utility function. We assume that $K_{1}<K_{2}$ and that all individuals are endowed with one unit of labor-that is, $L_{i}=1, i=1,2$. The utility function of an individual of type $i$ depends on the amounts of the private good-say, peanuts - and on the per capita level of public provision of peanuts in the nation $\bar{y}$. These preferences are assumed to be of the form:

$$
U_{i}(x, \bar{y})=U(x, \bar{y})= \begin{cases}x & \bar{y} \geq \hat{y} \\ -\infty & \bar{y}<\hat{y} .\end{cases}
$$

The welfare-state mindedness of the people is captured by the parameter $\hat{y}$. People cannot tolerate living in a society where the government does not guarantee a minimum level of peanuts. This may be because people experience extreme disutility when they see the miserably poor around them or because poverty carries the germ of negative externalities such as crime. But once this minimum level of peanuts is supplied, individuals' preferences are egoistic and well-behaved as in standard economic models.

There is a publicly available technology, $F: \mathbb{R}_{+}^{2} \rightarrow \mathbb{R}_{+}$, that transforms capital and labor inputs into peanuts. The production function $F$ is assumed to be concave, exhibits constant returns to scale, and satisfies $\lim _{K \rightarrow 0} \partial F /$ $\partial K(K, 1)=\infty, \lim _{K \rightarrow \infty} \partial F / \partial K(K, 1)=0$, and, for all $K>0, \partial F / \partial K(K, 1)>0$ and $\partial^{2} F / \partial K^{2}(K, 1)<0$. Given the constant returns to scale technology $F$, we can define the per capita production function $f(k)=F(k, 1)$. We assume throughout that $f\left(K_{1}\right)>\hat{y}$-in other words, that it is feasible to guarantee a level $\hat{y}$ even in a country populated only by type- 1 individuals.

Definition 1: An economy is a vector $\lambda:=\left(\lambda_{1}, \lambda_{2}\right) \in \mathbb{R}_{+}^{T}, \lambda \neq 0$.

Here $\lambda$ should be interpreted as the economy's population profile; that is, $\lambda_{i}$ is the measure of individuals of type $i$ in the economy. We denote by $k(\lambda)$ the capital-labor ratio of $\lambda$; thus, $k(\lambda)=$ $\sum_{i \in T} K_{i} \lambda_{i} / \sum_{i \in T} \lambda_{i}$. Since we concentrate on Walrasian allocations, which assign identical bundles to identical agents, we can confine ourselves to the kind of allocations defined below. 
Definition 2: An equal treatment allocation (or simply an allocation) for $\lambda$ is a vector of bundles $\left(x_{1}, x_{2}\right)$ such that

$$
\sum_{i \in T} x_{i} \lambda_{i} \leq f[k(\lambda)] \sum_{i \in T} \lambda_{i}
$$

An allocation is a description of a consumption plan for each type in the economy that is consistent with the resources of the economy.

Next we define a specific allocation for each economy which is associated with a price equilibrium and in which peanuts are partially provided by the government. For this purpose we assume that a level $\bar{y} \geq 0$ of peanuts is publicly provided to each individual in the economy and that it is financed by means of proportional income taxation. Hence, the government sets an amount $\bar{y}$ to be provided to each individual and a fixed income tax rate $t$. The notion defined below describes the equilibrium outcome once the level $\bar{y}$ is already determined. The level of public provision of peanuts, however, will be endogenously determined when we consider the international equilibrium.

Definition 3: A national equilibrium of an economy $\lambda$ relative to a public provision $\bar{y} \geq 0$ is a system $\left\langle r, w, t,\left(x_{i}\right)_{i \in T}\right\rangle$ such that

1. $\left(x_{i}\right)_{i \in T}$ is an allocation

2. for all $i \in T$ with $\lambda_{i}>0, x_{i}$ solves the problem

$$
\max U(x, \bar{y}) \quad \text { s.t. } 0 \leq x \leq(1-t)\left[w+r K_{i}\right]+\bar{y}
$$

3. $r=f^{\prime}[k(\lambda)]$ and $w=f[k(\lambda)]-f^{\prime}[k(\lambda)] k(\lambda)$

4. $t \sum_{i \in T}\left(w+r K_{i}\right) \lambda_{i}=\bar{y} \sum_{i \in T} \lambda_{i}$.

The first condition is a feasibility requirement. The second condition says that each individual maximizes his utility given the tax rate, the level of public provision of peanuts set by the government, and his income. Condition 3 is the usual factors market clearing conditions. The last one is a balanced budget condition for the government: the tax rate is chosen so as to finance the public provision of peanuts exactly.

The interpretation is as follows: $r$ and $w$ are the market prices (in peanuts terms) of capital and labor, respectively. Given these prices, individuals choose the level $x_{i}$ of consumption of peanuts.

If $\hat{y} \leq \bar{y}$, there is a unique national equilibrium relative to $\bar{y}$, characterized by

$$
\begin{aligned}
r & =f^{\prime}[k(\lambda)] \\
w & =f[k(\lambda)]-f^{\prime}[k(\lambda)] k(\lambda) \\
x_{i} & =[1-\bar{y} / f(k(\lambda))]\left[f(k(\lambda))-f^{\prime}(k(\lambda))\left(k(\lambda)-K_{i}\right)\right]+\bar{y} \\
t & =\bar{y} / f(k(\lambda)) .
\end{aligned}
$$


In this equilibrium the input prices are equal to the marginal productivity of the total endowments in the economy. The individual's gross income in peanuts terms is $f(k(\lambda))-f^{\prime}(k(\lambda))\left(k(\lambda)-K_{i}\right)$, which is taxed at a rate of $\bar{y} / f(k(\lambda))$. Each agent then uses his net income to buy peanuts in addition to that provided by the government.

Note that the total production of peanuts is independent of the level $\bar{y}$ of public provision. This means that the tax is not distortionary. This follows from the fact that all factors of production are inelastically supplied.

If $0 \leq \bar{y}<\hat{y}$ then everybody's utility is $-\infty$ independently of the allocation; consequently all allocations are equilibrium allocations.

The level $\bar{y}$ of publicly provided peanuts measures the degree of government intervention in the economy. The higher the level of publicly provided peanuts, the higher the equilibrium tax rate and the larger the magnitude of income redistribution. In other words, within the economy, the higher $\bar{y}$, the more equal the distribution of income according to any index that satisfies Dalton's principle of transfers.

If $\left\langle r, w, t,\left(x_{i}\right)_{i \in T}\right\rangle$ is a national equilibrium relative to $\bar{y}$, then $\left(x_{i}\right)_{i \in T}$ is the corresponding national equilibrium allocation. Associated with a given national equilibrium allocation, there is a national equilibrium utility profile. In a national equilibrium, the utility of an individual of type $i$ depends only on his own initial endowment, on the capital-labor ratio of the economy, and on the level $\bar{y}$ of government intervention. We can define the equilibrium utility function as follows:

$$
V\left(k ; K_{i} ; \bar{y}\right)= \begin{cases}{[1-\bar{y} / f(k)]\left[f(k)-f^{\prime}(k)\left(k-K_{i}\right)\right]+\bar{y}} & \hat{y} \leq \bar{y} \leq f(k) \\ -\infty & \bar{y}<\hat{y} .\end{cases}
$$

The value $V\left(k ; K_{i} ; \bar{y}\right)$ is the utility level enjoyed by an individual with initial endowment $K_{i}$, in an economy with a capital-labor ratio of $k$ in a national equilibrium with respect to $\bar{y}$.

Remark 1: In a national equilibrium, the individual's utility depends on his own capital endowment and on the following two parameters: (i) the capital-labor ratio of the economy and (ii) the level $\bar{y}$ of government intervention. In particular, it does not depend on the population measure of the economy. Therefore, for utility calculation purposes, an economy in national equilibrium is completely characterized by its average capital endowment and the public provision of peanuts.

We shall call a pair $(\lambda, \bar{y})$ a constituted nation, meaning an economy $\lambda$ that is in a national equilibrium with respect to $\bar{y}$. The difference between an economy and a constituted nation is that the former is just a group of individuals while the latter is an organized economy with a redistributive policy. Since the individual's utility in a constituted nation is fully characterized by $k(\lambda)$ and $\bar{y}$, we can draw both types of individuals' indifference curves as shown in Figure 1. 


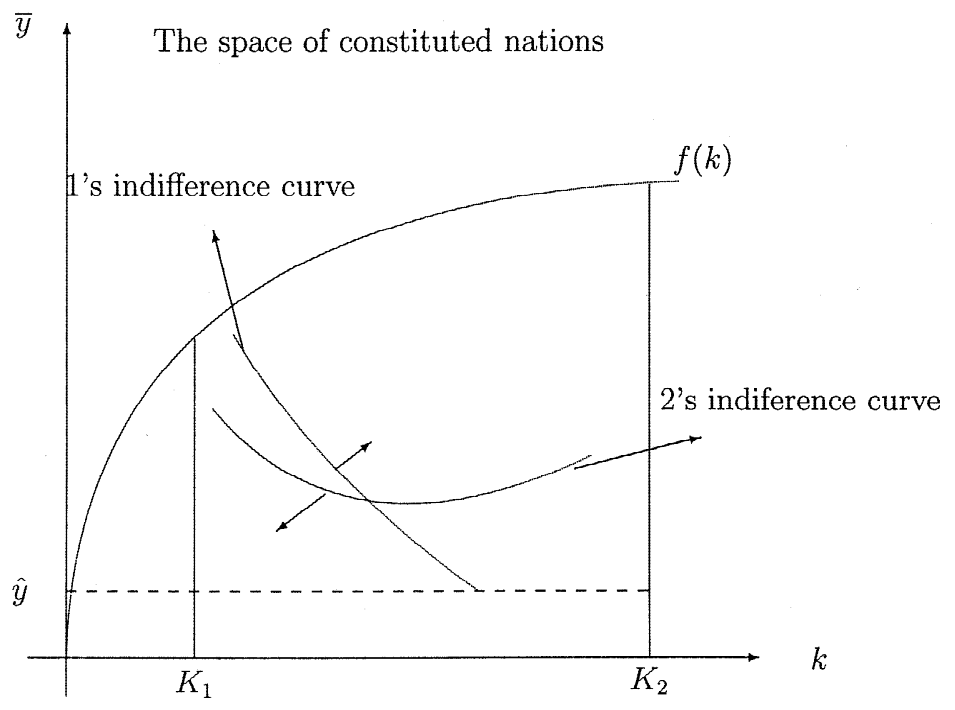

Figure 1: The space of constituted nations.

Note that all constituted nations are characterized by a capital-labor ratio between $K_{1}$ and $K_{2}$. Further note that the redistributive policies available to an economy $\lambda$ are characterized by $\bar{y} \leq f(k(\lambda))$. It can be checked that, when $\bar{y}>\hat{y}$, a type $i$ 's marginal rate of substitution of the redistributive instrument $\bar{y}$ for the capital-labor ratio $k$ is given by

$$
\frac{d \bar{y}}{d k}=-\frac{\bar{y}}{f^{\prime}(k)\left(k-K_{i}\right)}+\frac{\bar{y}}{f(k)}+\frac{\bar{y}(1-\bar{y} / f(k)) f^{\prime \prime}(k)}{f^{\prime}(k)} .
$$

Consequently, for type-1 individuals the slope of the indifference curve at each constituted nation is negative. Moreover the slope of the indifference curve of the rich is higher than that of the poor when evaluated at the same constituted nation.

\subsection{Analysis of the Function $V$}

We investigate the properties of the function $V$. The basic features of this function are summarized in Figure 2.

Given a tax rate $t$, an increase in the public provision of peanuts increases the utility of an individual of type $i$. On the other hand, the more peanuts that are publicly provided, the higher the equilibrium tax rate, which in turn lowers the after-tax income. As can easily be verified, 


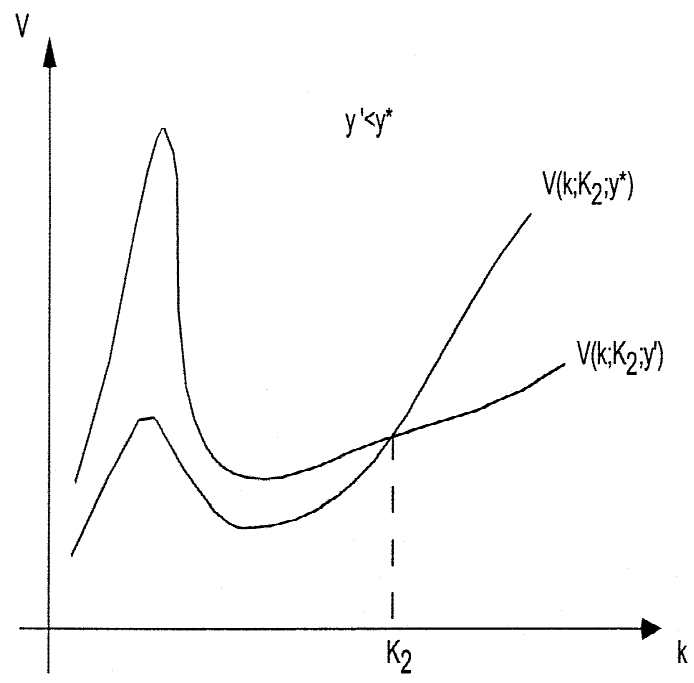

Figure 2: Properties of the function $V$.

the total effect depends on whether the agent's capital endowment is lower or higher than the economy's capital-labor ratio. If $k \geq K_{i}$, the higher the public provision of peanuts, the higher his equilibrium utility; and vice versa, if his capital endowment is higher than the economy's capital-labor ratio, the higher $\bar{y}$ the lower his equilibrium utility.

Second, $V$ is clearly increasing in the own capital endowment, since it affects gross income positively without influencing the tax rate. Finally, a change in the economy's capital-labor ratio brings about two effects. On the one hand, an increase in the capital-labor ratio reduces the equilibrium price of capital, thereby increasing the gross income of those with a relatively low capital endowment and decreasing gross income of those with high capital endowment. On the other hand, the same increase in the average capital endowment reduces the equilibrium tax rate, which increases the net income for any given level of gross income. Therefore, an increase in $k$ strictly increases the utility of those with lower-thanaverage capital-labor ratio but has an ambiguous effect on the utility of those with relatively high capital endowment.

This ambiguous effect is the driving force behind the results of this model. There are two forces in this model: one tends to unite people and the other tends to divide them. Subsidizing peanuts for the poor out of taxes paid by the rich is a force that can drive the rich to secede. The benefits from trade that emerge when two different types trade tend to unite them. The outcome of this tension between subsidization and benefit from trade is the main topic of this paper. 


\section{International Equilibrium}

Up to this point we have described the equilibrium in a given economy with a given level of public provision of peanuts. In this section we present and analyze the notion of international equilibrium, in which different national economies are endogenously formed. The level of public provision of peanuts in each of these national economies is also endogenously determined in the international equilibrium. Under our international equilibrium notion, nations are allowed to form provided they abide by the constraint that their public provision of peanuts is financed by means of a proportional income tax. An international equilibrium consists of an endogenously formed partition of the world economy into several constituted nations, each having a government that provides peanuts and finances it via proportional income tax. Moreover, no group of individuals can benefit from establishing a new constituted nation.

Let $T$ be the set of types as defined in Section 2. An economy $\mu \in \mathbb{R}_{+}^{T}$ is said to be a world economy if $\mu_{i}>0$ for all $i \in T$. That is, both types of individuals are present in the world economy.

Our equilibrium notion requires from a partition of the world economy that it not be improved upon by any potential nation:

Definition 4: An international equilibrium of the world economy $\mu$ is a finite collection of constituted nations $\left\langle\left(\lambda^{1}, \bar{y}^{1}\right), \ldots,\left(\lambda^{m}, \bar{y}^{m}\right)\right\rangle$ such that:

(i) $\mu=\sum_{j=1}^{n} \lambda^{j}$;

(ii) there is no constituted nation $\left(\sigma, y^{\prime}\right)$ such that for all $i \in T$ with $\sigma_{i}>0$ there is a nation $\lambda^{j}$ with $\lambda_{i}^{j}>0$ and $V\left(k(\sigma), K_{i}, y^{\prime}\right)>$ $V\left(k\left(\lambda^{j}\right), K_{i}, \bar{y}^{j}\right)$.

Condition (i) says that the inhabitants of all the constituted nations add up to the inhabitants of the world. Condition (ii) says that no group of individuals can improve upon the existing partition of the world by forming their own constituted nation. It is implicitly assumed in this condition that no capital mobility between constituted nations is allowed. Each constituted nation, existing or potential, must consume an amount of peanuts that can be produced with its residents' resources. The case of perfect capital mobility is briefly analyzed in Section 4.3.

In order to improve upon a given partition of the world economy it must be possible to form a new constituted nation by picking individuals from the partition nations in such a way that all the individuals in the new nation are better off.

According to this definition, a partition of the world economy into nations is in equilibrium if it is immune to unions of existing nations, secessions of regions from existing countries, or unions of regions of different nations. 
Remark 2: Since the equilibrium utility function of an individual does not depend on the population measure of his country we can split up any equilibrium nation into several nations with the same capitallabor ratio and still remain in equilibrium, and vice versa. Therefore, any equilibrium partition is equivalent to another equilibrium partition in which all the nations with the same average capital endowment in the first equilibrium constitute a single nation in the second equilibrium.

\section{Characterization of the International Equilibrium}

In this section we characterize the set of international equilibria and show their existence under a mild assumption which guarantees that for each world economy the international equilibrium is essentially unique. We also give an informal description of the equilibria.

The lemma below and its corollary show that in the analysis of the international equilibria it is sufficient to restrict attention to constituted nations with $\bar{y}=\hat{y}$.

LEMMA 1. Let $(\lambda, \bar{y})$ be a constituted nation with $\lambda \gg 0$ and $\bar{y}>\hat{y}$. There is an economy $\sigma$ such that for all $i \in T$ with $\sigma_{i}>0, V\left(k(\sigma), K_{i}, \hat{y}\right)>V\left(k(\lambda), K_{i}, \bar{y}\right)$.

Proof: Consider a nation $\lambda$ with a positive measure of individuals of both types where the public provision of peanuts is given by $\bar{y}>\hat{y}$. It follows from the difference in the marginal rates of substitution of the two types of individuals (Equation (1)) that there is a combination $(k, y)$ of capital-labor ratio and public provision of peanuts to the southeast of $(k(\lambda), \bar{y})$ that increases the indirect utility of both types (see Figure 1). Since this is true for any interior point of the space of constituted nations, there must exist a path from the initial $(k(\lambda), \bar{y})$ to the boundary of the box. If the path reaches $\hat{y}$, we are done. If it hits $K_{2}$ then, since the boundary nation contains only rich type citizens, we can continue the path down to $\hat{y}$ without changing the individuals' utilities.

Lemma 1 states that a mixed nation whose government provides more than $\hat{y}$ peanuts per capita is not stable because a subgroup of individuals can secede and form a new constituted nation that provides exactly $\hat{y}$, making all its citizens better off. The instability of a nation that provides more than the equilibrium level cannot be remedied by setting up the "appropriate" institution to determine the level of income distribution. For example, a system where the level of $\bar{y}$ is determined by majority voting will lead to an unstable outcome unless the result is $\hat{y}$. There will always be pressure for secession if the chosen redistribution level (determined by whatever mechanism) is not the equilibrium one. 
COROLLARY 1: Let $\left\langle\left(\lambda^{1}, \bar{y}^{1}\right), \ldots,\left(\lambda^{m}, \bar{y}^{m}\right)\right\rangle$ be an international equilibrium. Then for all $i=1, \ldots, m$, if $\lambda_{1}^{i}>0$ and $\lambda_{2}^{i}>0$ then $\bar{y}^{i}=\hat{y}$. Otherwise, $\bar{y}^{i} \geq \hat{y}$.

Proof: It is clear that no nation $\lambda^{i}$ in an international equilibrium can have a public provision of peanuts lower than $\hat{y}$ since by increasing $\bar{y}^{i}$ to $\hat{y}$ the utility level of everybody increases. By the previous lemma, a strictly mixed equilibrium nation cannot publicly provide more than $\hat{y}$ peanuts per capita, as a subset of individuals in the nation can improve upon that level.

Corollary 1 says that in an international equilibrium, in all nations that have a mixed population the government provides the same amount of peanuts per capita, $\hat{y}$. Nations with inhabitants of only one type may provide more than $\hat{y}$ in equilibrium, but because actual transfers are null, these equilibria are utility equivalent to those where the government provides exactly $\hat{y}$.

The above results are useful because they allow us to restrict attention to nations that provide $\hat{y}$ peanuts per capita when we look for an international equilibrium. Corollary 1 tells us that the equilibrium nations provide exactly $\hat{y}$, and Lemma 1 tells us that in order to check whether a given partition of the world into nations is an international equilibrium it is enough to check for deviations where the deviating nations have $\hat{y}$ as the redistributive policy. The reason is that if a nation can improve upon a given partition using some $\bar{y}$ as the redistributive policy instrument, then Lemma 1 guarantees that there is another nation (one that secedes from the deviating one) that improves upon the same partition with $\hat{y}$ as the redistributive instrument. Consequently, from now on we restrict attention to nations that provide the endogenously determined level $\bar{y}=\hat{y}$ of peanuts per capita.

Let $\mu$ be a world economy. The following sets are used in the definitions below: For each $\tilde{k} \in\left[K_{1}, K_{2}\right]$ and $\bar{y} \geq \hat{y}$ let

$$
X(\tilde{k}, \bar{y})=\left\{k \in\left[\tilde{k}, K_{2}\right] \mid V\left(k, K_{2}, \bar{y}\right) \geq V\left(z, K_{2}, \bar{y}\right), \forall z \in\left[\tilde{k}, K_{2}\right]\right\} .
$$

The set $X(\tilde{k}, \bar{y})$ is the set of maximizers of the utility of type-2 individuals in the interval $\left[\tilde{k}, K_{2}\right]$ given the redistributive policy $\bar{y}$. Since $V$ is continuous, $X(\tilde{k}, \bar{y}) \neq \varnothing$ for all $\tilde{k} \in\left[K_{1}, K_{2}\right]$ and for all $\bar{y} \geq \hat{y}$.

Definition 5: Let $\mu$ be a world economy. It is a well-behaved economy if for all $\tilde{k} \in\left[K_{1}, K_{2}\right]$, the set $X(\tilde{k}, \hat{y})$ is a singleton or is $\left\{\tilde{k}, K_{2}\right\}$.

This definition requires that the equilibrium utility of type-2 individuals should have either one or two maximizers in the interval $\left[\tilde{k}, K_{2}\right]$. But in the latter case, the maximizers should be the extreme points of the interval. Although we cannot guarantee that for all production functions all economies are well-behaved, we could not find any counterexample. 
We now give an informal description of the equilibria of a wellbehaved world economy. Those who are interested only in the formal description of the equilibrium can skip to the next subsection.

\subsection{Informal Description of the Equilibria}

For each world economy, all equilibria are equivalent in the sense that they induce the same distribution of consumption plans. Moreover, for any capital-labor ratio $k$, the measure of individuals living in a nation whose capital-labor ratio is $k$ is the same for all equilibria. They can differ only in the number, and therefore size, of nations with a capital-labor ratio $k$.

The economies can be divided into four cases:

Case 1: A nation populated only by type-2 individuals is preferred by the rich to any other possible nation: $K_{2} \in X\left(K_{1}, \hat{y}\right)$.

Case 2: A nation populated only by type-2 individuals is preferred by the rich to any nation with more than the world capital-labor ratio but there are poorer nations that are preferred: $K_{2} \notin$ $X\left(K_{1}, \hat{y}\right)$ and $\left\{K_{2}\right\}=X(k(\mu), \hat{y})$.

Case 3: A nation with the world capital-labor ratio is preferred by the rich to any nation with more than the world capital-labor ratio: $K_{2} \notin X\left(K_{1}, \hat{y}\right)$ and $k(\mu) \in X(k(\mu), \hat{y})$.

Case 4: The nation most preferred by the rich has a capital-labor ratio strictly between the world capital-labor ratio and $K_{2}: K_{2} \notin$ $X\left(K_{1}, \hat{y}\right)$ and $\left\{k(\mu), K_{2}\right\} \cap X(k(\mu), \hat{y})=\varnothing$.

The four cases are illustrated in Figure 3. In Case 1 there are no mixed nations in equilibrium. Note that any partition of the world economy that contains a mixed nation is improved upon by an economy that consists of only type-2 individuals. In Case 2 all international equilibrium partitions consist of two types of nations: a rich nation containing only type-2 individuals and a poor nation with a mixed population and with a capital-labor ratio that makes its type-2 inhabitants indifferent between staying in their own nation and migrating to another existing nation. Note that all type-1 individuals live in the poor nation(s) and would like to migrate to a richer nation, but are prevented from doing so by the immigration "services" of the rich nations. In Case 3, all international equilibria consist of nations with the same capital-labor ratio (the world's capitallabor ratio). Unlike the previous one, in this case no one wants to migrate to any existing nation. This fact, however, has nothing to do with our equilibrium concept-rather, what makes this an equilibrium is that any potential nation that type-1 individuals would like to live in needs some type-2 individuals, but no type-2 individuals will agree to form such a nation. (Or similarly, any potential nation that type-2 individuals would like to live in needs some type-1 individuals, but the latter will not agree 

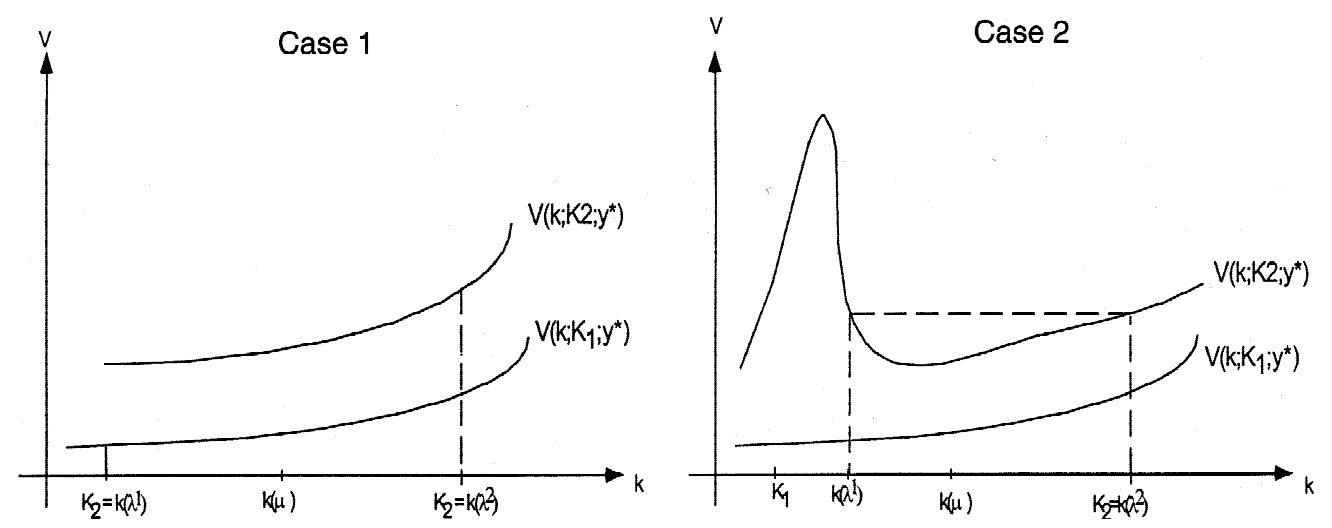

1
0
3
3
0
0
0
0
0
7
7
0.0
0
0
0
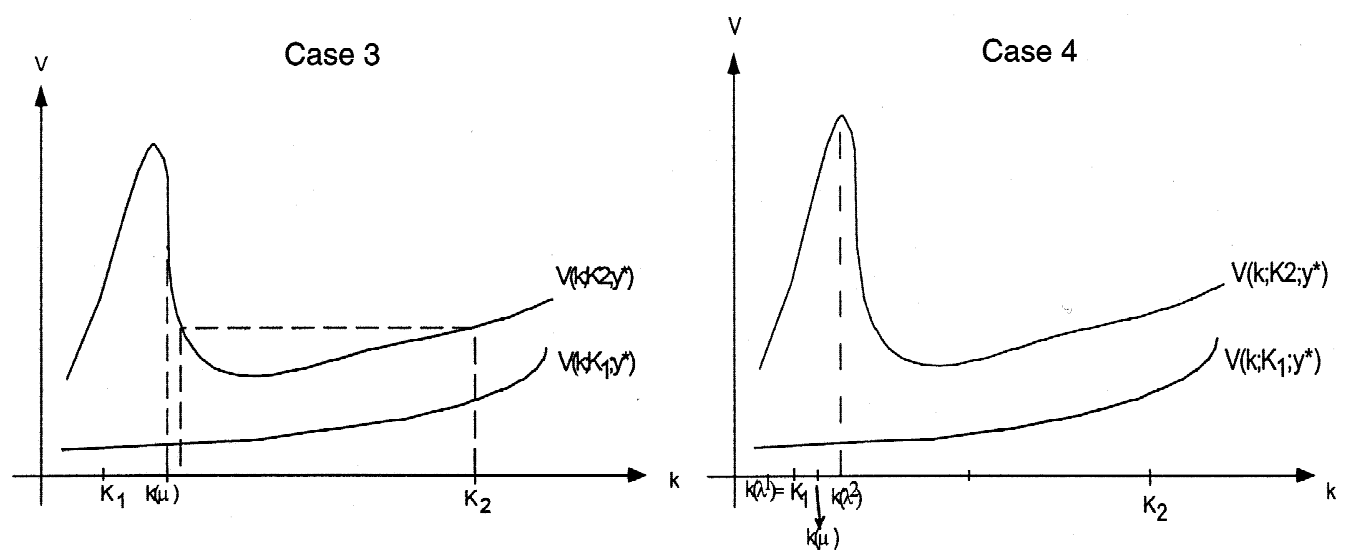

Figure 3: Four world economies. 
to cooperate in the formation of such a nation.) Note that in all three cases, all the individuals of the same type have the same consumption plan.

In the last case all the international equilibrium partitions consist of two types of nations: a poor nation containing only type-1 individuals and a richer nation with a mixed population and with the optimal capitallabor ratio from the point of view of type-2 individuals. Note that in this type of equilibrium all type-2 individuals have the same utility level. This is not the case, however, for type-1 individuals. Those who live in richer countries enjoy a higher utility level than those living in poorer ones. Note that Cases 2, 3, and 4 may correspond to three world economies that differ only in the proportions of type-1 and type-2 individuals, and consequently in their respective capital-labor ratios. In Case 2 there is a high proportion of type-2 individuals, in Case 3 this proportion is lower, and in Case 4 this proportion is the lowest. Correspondingly, a type-2 individual's equilibrium utility improves from Case 2 to Case 3 and from Case 3 to Case 4. This follows from the fact that in Cases 2 and 3, the competition among type-2 individuals prevents them from getting the optimal utility level.

\subsection{Formal Characterization}

Let $\hat{k}=\max X\left(K_{1}, \hat{y}\right)$; that is, $\hat{k}$ is the highest capital-labor ratio among those that maximize the equilibrium utility of type-2 individuals in $\left[K_{1}, K_{2}\right]$. (The continuity of $V$ implies that $\hat{k}$ is well-defined.)

Define

$$
B=X(k(\mu), \hat{y})
$$

and let $V^{*}=V\left(k, K_{2}, \hat{y}\right)$ for some $k \in B . V^{*}$ is the maximum utility level that type-2 individuals can get in a nation characterized by a capital-labor ratio between $k(\mu)$ and $K_{2}$. Let

$$
C= \begin{cases}\left\{K_{1}\right\} & \text { if } \hat{k} \geq k(\mu) \\ \left\{k \in[\hat{k}, k(\mu)]: V\left(k, K_{2}, \hat{y}\right)=V^{*}, \text { and } k \in X(k, \hat{y})\right\} & \hat{k}<k(\mu) .\end{cases}
$$

Since $V\left(\hat{k}, K_{2}, \hat{y}\right) \geq V^{*}$ and $V\left(k(\mu), K_{2}, \hat{y}\right) \leq V^{*}$, by the mean value theorem $C \neq \varnothing$. The sets $B$ and $C$ will play an important role in the characterization of the international equilibria. Theorem 1 below states that equilibrium nations have capital-labor ratios belonging to $B$ or $C$. Nations that are richer than the average will have a capital-labor ratio in $B$ and poorer than average nations will have a capital-labor ratio in $C$.

Remark 3: Since Corollary 1 says that all the equilibrium nations publicly provide $\hat{y}$ peanuts, we shall denote a generic international equilibrium by the partition of the world into economies, without writing the distributive policies-that is, by $\mathcal{E}=\left\langle\lambda^{1}, \ldots, \lambda^{m}\right\rangle$. Moreover, when we 
say that a certain economy can improve upon a partition we mean that the economy can improve upon by applying the distributive policy $\hat{y}$. Thus we take advantage of Lemma 1, which allows us to restrict attention to this kind of improvements.

We are now ready to state the main theorem of this section.

THEOREM 1: Let $\mu$ be a well-behaved world economy and let $\mathcal{E}=\left\langle\lambda^{1}, \ldots, \lambda^{m}\right\rangle$ be a partition of $\mu . \mathcal{E}$ is an international equilibrium if and only if for all $j=$ $1, \ldots, m, k\left(\lambda^{j}\right) \in B \cup C$.

Theorem proofs are in the Appendix.

COROLLARY 2: Let $\mathcal{E}$ be an international equilibrium of the world economy $\mu$. Then all type-2 individuals get the same utility level.

Proof: All equilibrium nations with some type- 2 individuals in it have a capital-labor ratio in $B \cup C$, thus providing them with a utility level of $V^{*}$.

We now turn to the question of uniqueness of the equilibrium. Let $\mathcal{E}=\left\langle\lambda^{1}, \ldots, \lambda^{m}\right\rangle$ be an international equilibrium. Let $k(\mathcal{E})=\left\{k_{1}, \ldots, k_{n}\right\}$ be the set of capital-labor ratios of the equilibrium nations. That is, $k_{j} \in k(\mathcal{E})$ is the capital-labor ratio of some $\lambda^{i} \in \mathcal{E}$. For each $k_{j} \in k(\mathcal{E})$ define the following economy:

$$
\sigma^{j}=\sum_{k\left(\lambda^{i}\right)=k_{j}} \lambda^{i}
$$

The economy $\sigma^{j}$ is the one that results from the integration of all the equilibrium nations with a capital-labor ratio of $k_{j}$.

Let $\mathcal{E}^{\prime}=\left\langle\sigma^{1}, \ldots, \sigma^{n}\right\rangle$ be the partition of the world economy that results from this process of integration. Call it the coarsest equilibrium partition of $\mu$ relative to $\mathcal{E}$. Note that by Theorem $1 \mathcal{E}^{\prime}$ is an equilibrium. We say that $\mu$ has an essentially unique equilibrium if all its equilibria have the same coarsest partition of $\mu$.

THEOREM 2: Every well-behaved economy $\mu$ has an essentially unique equilibrium.

\subsection{The Case of Perfect Capital Mobility}

The concept of international equilibrium we have worked with so far assumes implicitly that capital is immobile. Namely, an individual's capital must be employed in the nation where he lives. In this subsection we discuss the case where capital is perfectly mobile across nations.

Consider a situation where there is an international capital market in which capital is exchanged for peanuts. As before, labor is internationally immobile. Further assume that an individual pays (capital and labor) income tax only in the nation he belongs to. Since capital is perfectly mobile, in 
equilibrium its international price will be $f^{\prime}[k(\mu)]$, where $\mu$ denotes the world economy. Further, there will be price equalization in the national labor markets, with a wage rate that corresponds to the world capital-labor ratio. An international equilibrium with perfect capital mobility will be a partition of the world economy into nations such that there is no group of individuals that can benefit by forming its own nation which still participates in the international capital market.

Since equilibrium prices are independent of the partition of the world into nations, it follows that there cannot be a nation that has a positive measure of both type- 1 and type- 2 individuals. To see this, note that type-2 individuals can improve upon a nation like this by seceding and forming a nation of their own, thus avoiding the subsidization of the poor. Consequently, the essentially unique equilibrium is a fully segregated world consisting of one-type nations. The resulting allocation will be Pareto optimal due to the perfect capital mobility. However, there will be no effective income redistribution, no matter the level of welfare-state mindedness of the individuals. In fact, the equilibrium allocation will be identical to the one that results in a world with no welfare-state mindedness and no capital mobility.

In the next section it is shown that without capital mobility a redistribution of income occurs and its level depends on the degree of welfarestate mindedness.

\section{Welfare-State Mindedness, Integration, and Income Distribution}

In this section we make some comparative-statics analyses with respect to the welfare-state mindedness, measured by the minimum level of peanuts publicly provided that an individual can tolerate. It is clear that in a given nation, the more peanuts are publicly provided, the more equal the resulting distribution of income. On the other hand a higher level of welfarestate mindedness may change the composition of the equilibrium nations. Therefore, it is not clear how a higher degree of welfare-state mindedness affects the distribution of income in the world economy.

We first find a range of levels of welfare-state mindedness in which the corresponding international equilibria yield only nations with the same capital-labor ratio: $k(\mu)$. Namely, for different levels of welfare-state mindedness within this range, the internal composition of the equilibrium nations is the same. As a result, within this range an increase in the level of welfare-state mindedness yields a worldwide decrease in income inequality. Outside this range, however, changes in the level of welfare-state mindedness do change the internal composition of nations and their capital-labor ratios, and consequently their effect on income distribution is not obvious. 
The following lemma states necessary and sufficient conditions for an international equilibrium to yield the whole world as one nation as the essentially unique equilibrium. ${ }^{1}$

LEMMA 2: Let $\mu$ be a world economy. Let $\mathcal{E}=\left\langle\lambda^{1}, \ldots, \lambda^{m}\right\rangle$ be an international equilibrium. For all $j=1, \ldots, m, k\left(\lambda^{j}\right)=k(\mu)$ if and only if

$$
k(\mu) \in X(k(\mu), \hat{y}) .
$$

Proof: Let $\mathcal{E}=\left\langle\lambda^{1}, \ldots, \lambda^{m}\right\rangle$ be an international equilibrium. Assume that $k\left(\lambda^{j}\right)=k(\mu)$ for all $j=1, \ldots, m$. Since the indirect utility function $V$ of type- 1 individuals is strictly increasing in $k$ within $\left[K_{1}, K_{2}\right]$, all type-1 individuals would like to join a nation with a capital-labor ratio larger than $k(\mu)$. Since $\mathcal{E}$ is an international equilibrium, it must be that no type- 2 individual wants to join such a nation. Thus, condition (4) holds.

Assume now that condition (4) holds. Therefore $k(\mu) \in B$ and, by Theorems 1 and 2, if $\lambda$ is an equilibrium nation it must be that $k(\lambda)=$ $k(\mu)$.

In terms of our informal description of the equilibria, Lemma 2 states that the whole world as a nation is an equilibrium when the parameters of the economy correspond to Case 3. Since our model makes no prediction about the number and size of nations, all the equilibria that satisfy condition (4) are equivalent, in terms of the consumption plans, to the international equilibrium in which the whole world is one nation.

Let $\mu$ be a world economy and let

$$
\mathcal{A}=\{\hat{y} \in[0, f(k(\mu))]: \text { condition (4) is satisfied }\}
$$

The set $\mathcal{A}$ is the range of levels of welfare-state mindedness that result in equilibrium nations with the same proportions of type-1 and type-2 individuals. We are interested in the effect on worldwide income distribution when the economies are outside this range.

Define $\breve{y}$ as the maximum element of $\mathcal{A} .^{2}$ Welfare-state mindedness levels above $\breve{y}$ are outside the range that results in the whole world as one nation being essentially the unique equilibrium. Restricting attention to levels above $\breve{y}$, we would like to check how changes in the welfare-state mindedness affect the worldwide distribution of income.

In order to answer this question, we need to compare world economies that differ only in the welfare-state mindedness of the individuals.

\footnotetext{
${ }^{1}$ Recall that, in accordance with Remark 3, we omit the $\hat{y}$ s from the equilibrium nations and that by "improve upon" we mean improving upon applying the distributive policy $\hat{y}$.

${ }^{2} \mathcal{A}$ is clearly nonempty since, as can be easily verified, $0 \in \mathcal{A}$. Moreover, since $\mathcal{A}$ is a closed subset of a bounded set, $\mathcal{A}$ is compact. Therefore, $\breve{y}$ is well defined.
} 
For this reason in this section a world economy will be denoted by $\mu[\hat{y}]$ where $\hat{y}$ denotes the parameter of the utility function of the people in that world.

Theorem 3 below states that in a large class of economies if the level of welfare-state mindedness is outside $\mathcal{A}$, namely if there are different nations in equilibrium, an increase in the level of welfare-state mindedness results in an increase in worldwide income inequality and at the same time no individual consumes more peanuts. This large class of economies is given by the following definition.

Definition 6: Let $y^{*} \geq \hat{y}$ be a level of public provision of peanuts. The world economy $\mu[\hat{y}]$ is U-shaped with respect to $y^{*}$ if the function $V\left(\cdot, K_{2}, y^{*}\right)$ has no local maximum in the interval $\left(k(\mu), K_{2}\right)$.

Given that $V\left(k, K_{2}, y^{*}\right)$ is increasing at $k=K_{2}$, this assumption means that $V$ is either increasing or U-shaped in the interval $\left(k(\mu), K_{2}\right)$. It can be shown that if $\mu[\hat{y}]$ is U-shaped with respect to $y^{*}, V\left(k, K_{2}, \bar{y}\right)$ has no local maximum in $\left(k(\mu), K_{2}\right)$ for any $\bar{y} \in\left[\hat{y}, y^{*}\right]$.

We can now state the main theorem of this section.

THEOREM 3: Let $\tilde{y} \geq \max \mathcal{A}$ and let $y^{*}>\tilde{y}$. Assume that $\mu\left[y^{*}\right]$ and $\mu[\tilde{y}]$ are well-behaved, $U$-shaped economies with respect to $y^{*}$ and $\tilde{y}$, respectively. Choose an international equilibrium of $\mu[\tilde{y}]$ and denote it by $\tilde{\mathcal{E}}$. Assume further that there is an equilibrium nation in $\tilde{\mathcal{E}}$ with a positive measure of each type of individuals. Similarly, denote by $\mathcal{E}^{*}$ an international equilibrium of $\mu\left[y^{*}\right]$. The world distribution of income under $\tilde{\mathcal{E}}$ is Lorenz superior to the world distribution of income under $\mathcal{E}^{*}$. Moreover, in $\tilde{\mathcal{E}}$ all type-1 individuals consume more peanuts than in $\mathcal{E}^{*}$ and all type-2 individuals consume no less.

In terms of our informal description of the equilibria, Theorem 3 tells us that when we compare an equilibrium that corresponds to Case 2 to one that corresponds to a higher level of welfare-state mindedness, the equilibrium outcome of the former Lorenz and Pareto dominates the equilibrium outcome of the latter.

This theorem says that the pursuit of a more vigorous redistributive policy does not necessarily reduce worldwide inequality. Moreover, there is an interval of parameter values of welfare-state mindedness such that the equilibrium income distribution of the world is less equal the more welfare-state minded the people are. The point is that any attempt to redistribute more than what is implicit in the public provision of $\tilde{y}$ results in some type-2 individuals fleeing from the poor nations to the rich nations. This in turn results in an efficiency loss that is suffered only by type-1 individuals.

If one wants the redistributive policy not to increase income inequality and not to damage the welfare of the poor, it is necessary that this 
policy be within the set $\mathcal{A}$. One can interpret this result to mean that a necessary condition for a stable economic union among countries with different wealth levels is that there should not be an overly strong redistributive policy. The implication of this result to the future of the European Union is clear.

\section{Related Literature}

The related literature can be traced back to the seminal paper by Tiebout (1956). (For a good appraisal of this work, see Kurz 1994). Tiebout conjectured that in a local public goods economy there exist equilibria and all of them are Pareto optimal. Two kinds of models have tried to formalize Tiebout's conjecture; they differ in the interpretation of the equilibrium he had in mind.

The first interpretation is that of a Nash equilibrium. Here an equilibrium is a partition of the agents into an exogenously fixed number of jurisdictions such that no individual strictly prefers some other jurisdiction to the one he belongs to. Each jurisdiction produces a local public good that is financed by taxing its residents. The assumption of free mobility between jurisdictions is implicit in the solution concept. Along with this interpretation, Westhoff (1977) gives sufficient conditions for existence of equilibrium in an economy with an exogenously given number of jurisdictions. Bewley (1981) gives several examples of economies where this kind of Tiebout equilibrium either does not exist or is not efficient. Benabou (1993) applies this equilibrium concept to model the population composition of different neighborhoods in a city. The analysis of free mobility as a constraint to redistribution of income is the topic of Epple and Romer (1991). Their model, however, takes the number of jurisdictions within a federation as exogenously given. More recently, Bolton and Roland (1997) present a political economy analysis of secession in a setup very similar to ours, but where two regions in a nation are exogenously given.

The second interpretation of Tiebout's equilibrium identifies it with the core. According to this interpretation, an endogenous partition of the economy is an equilibrium if no group of individuals can make each of its members better off by forming a new jurisdiction that provides the local public good. Along with this second interpretation, early contributions include Wooders (1978), Bennett and Wooders (1979), Guesnerie and Oddou (1981), and Greenberg and Weber (1986). Some recent contributions include Conley and Wooders (1997), Cole and Prescott (1997), and Ellickson et al. (1999). According to this view, migration tensions may prevail in equilibrium. Namely, it may well be the case that some group of individuals would prefer to migrate to an existing jurisdiction. In this sense it is appropriate to call the equilibrium jurisdictions 
"nations." 3 On the other hand, Greenberg and Weber showed that their equilibrium is also a Nash equilibrium or an equilibrium with free mobility. Therefore, in their equilibrium there are no migration tensions. Alesina and Spolaore (1997) build a model similar to the one in Guesnerie and Oddou and in Greenberg and Weber. The main difference lies in the solution concept applied. The latter papers apply the core, but Alesina and Spolaore apply a hybrid solution concept that mixes both interpretations of Tiebout's equilibrium. In their model a coalition can improve upon a partition of the economy only if it proposes another partition, which is stable in a dynamic sense, in which nobody wants to migrate to a neighboring country and which improves the welfare of some majority of each of the deviating jurisdictions. Since their solution concept requires that no individual should want to migrate to any existing jurisdiction, migration tensions are absent by definition.

With the exceptions of Epple and Romer (1991) and Bolton and Roland (1997), our model differs from all the above in that it focuses on the distributive role of taxation instead of on the provision of public goods among agents with different preferences. We adopt the wellestablished solution concept of the core. Contrary to Greenberg and Weber (1986), we get that in equilibrium there may exist migration tensions. Moreover, when the equilibrium number of distinct nations is greater than one, migration tensions do appear: poor individuals always want to migrate to richer nations. Another important difference resides in the fact that although in Westhoff (1977) and in Greenberg and Weber, individuals with similar characteristics cluster together in equilibrium (in Alesina and Spolaore 1997 this feature is imposed rather than derived as an equilibrium phenomenon), in our model, as in Bennett and Wooders (1979), an equilibrium may consist of nations, some of which have several types of individuals. The reason is that in the latter there are gains from trade only when traders differ from each other.

Our paper is related and complements the macroeconomic literature on migration. In a static model of migration, Razin and Sadka (1995) show that when a country's individuals differ only in their cost of becoming skilled laborers but not in their initial endowments, migration constitutes a Pareto improvement. If the government applies a proportional income tax, however, and redistributes revenues equally to the entire population (regardless of their national status), migration is utilityreducing for the native-born. This result coincides with our observation that the use of taxation for redistributive purposes may cause incentives to set barriers to migration. Our model formalizes the creation of such barriers.

\footnotetext{
${ }^{3}$ One may say that if a core allocation can be supported by prices then, given these prices, there are not migration tensions. But still, positive prices are a manifestation and a way to deal with the migration tensions.
} 


\section{Appendix: Proofs of the Theorems}

The following lemma will be very useful in proving the theorems.

LEMMA 3: Let $\mu=\left(\mu_{1}, \mu_{2}\right)$ be an economy with average capital endowment $k(\mu)$. Let $\underline{k}$ and $\bar{k}$ be two arbitrary numbers satisfying $K_{1} \leq \underline{k}<k(\mu)<\bar{k} \leq K_{2}$. Then, there exists a unique partition of the economy $\mu$ into two economies, $\lambda$ and $\tau$, such that $\lambda+\tau=\mu$ and $k(\lambda)=\underline{k}, k(\tau)=\bar{k}$.

Proof: In order to find the two economies we need to solve the following system of linear equations, check that there is a unique solution, and verify that it is nonnegative:

$$
\begin{aligned}
\lambda_{1}+\tau_{1} & =\mu_{1} \\
\lambda_{2}+\tau_{2} & =\mu_{2} \\
\left(\lambda_{1} K_{1}+\lambda_{2} K_{2}\right) /\left(\lambda_{1}+\lambda_{2}\right) & =\underline{k} \\
\left(\tau_{1} K_{1}+\tau_{2} K_{2}\right) /\left(\tau_{1}+\tau_{2}\right) & =\bar{k} .
\end{aligned}
$$

It can be shown that the solution to this system is as follows:

$$
\begin{aligned}
& \lambda_{1}=\left(\mu_{1}+\mu_{2}\right)\left(K_{2}-\underline{k}\right)(\bar{k}-k(\mu)) /\left[\left(K_{2}-K_{1}\right)(\bar{k}-\underline{k})\right] \\
& \lambda_{2}=\left(\mu_{1}+\mu_{2}\right)\left(\underline{k}-K_{1}\right)(\bar{k}-k(\mu)) /\left[\left(K_{2}-K_{1}\right)(\bar{k}-\underline{k})\right] \\
& \tau_{1}=\left(\mu_{1}+\mu_{2}\right)\left(K_{2}-\bar{k}\right)(k(\mu)-\underline{k}) /\left[\left(K_{2}-K_{1}\right)(\bar{k}-\underline{k})\right] \\
& \tau_{2}=\left(\mu_{1}+\mu_{2}\right)\left(\bar{k}-K_{1}\right)(k(\mu)-\underline{k}) /\left[\left(K_{2}-K_{1}\right)(\bar{k}-\underline{k})\right],
\end{aligned}
$$

which is nonnegative. This completes the proof of the lemma.

Lemma 3 implies that in an economy with a positive measure of individuals of both types, it is always feasible to form a new economy by choosing suitable proportions of individuals of each type, with an average capital endowment equal to any number between $K_{1}$ and $K_{2}$.

Proof of Theorem 1:

LEMMA 4: Let $\mathcal{E}=\left\langle\lambda^{1}, \ldots, \lambda^{m}\right\rangle$ be a partition of $\mu$ and assume that there exists $\lambda^{j} \in \mathcal{E}$ such that $k\left(\lambda^{j}\right) \geq k(\mu)$ and $k\left(\lambda^{j}\right) \notin B$. Then $\mathcal{E}$ is not an international equilibrium.

Proof of Lemma 4: Since $k\left(\lambda^{j}\right) \geq k(\mu)$, there is a positive measure of type-2 individuals in $\lambda^{j}$. Assume there exists a nation $\lambda^{i} \in \mathcal{E}$ with $k\left(\lambda^{i}\right)<k(\mu)$. Then, there is a positive measure of type-1 individuals in $\lambda^{i}$. Now, some suitable proportion of type-2 individuals of $\lambda^{j}$ and of type-1 individuals of $\lambda^{i}$ can form a nation $\sigma$ with $k(\sigma) \in$ $B$, thus improving upon $\mathcal{E}$. If there is no nation $\lambda^{i} \in \mathcal{E}$ with $k\left(\lambda^{i}\right)<k(\mu)$, it must be that $k\left(\lambda^{j}\right)=k(\mu) \notin B$. But then there is an economy $\sigma \in B$ that improves upon $\mathcal{E}$. 
Let $\mathcal{E}=\left\langle\lambda^{1}, \ldots, \lambda^{m}\right\rangle$ be an international equilibrium and assume by contradiction that there is $\lambda^{j} \in \mathcal{E}$ such that $k\left(\lambda^{j}\right) \notin B \cup C$. By Lemma $4, k\left(\lambda^{j}\right)<k(\mu)$. Then there exists another nation $\lambda^{i} \in \mathcal{E}$ with $k\left(\lambda^{i}\right)>k(\mu)$. Moreover, by Lemma $4, k\left(\lambda^{i}\right) \in B$.

Case 1: $\quad V\left(k\left(\lambda^{j}\right), K_{2}, \hat{y}\right)>V^{*}$. In this case, some type-1 individuals of $\lambda^{j}$ and some type-2 individuals of $\lambda^{i}$ can form a nation $\sigma$ with $k(\sigma)=k\left(\lambda^{j}\right)+\epsilon$ for $\epsilon>0$ small enough, which improves upon $\mathcal{E}$, contradicting the assumption that $\mathcal{E}$ is an equilibrium.

Case 2: $\quad V\left(k\left(\lambda^{j}\right), K_{2}, \hat{y}\right) \leq V^{*}$.

Case 2.1: $k(\mu) \leq \hat{k}$. In this case it must be that $V\left(\hat{k}, K_{2}, \hat{y}\right)=V^{*}$. Since $k\left(\lambda^{j}\right) \notin C, \lambda^{j}$ is a mixed nation. Since $\mu$ is a well-behaved economy, $V\left(k\left(\lambda^{j}\right), K_{2}, \hat{y}\right)<V^{*}$. Therefore some individuals of $\lambda^{j}$ can form a nation $\sigma$ with $k(\sigma)=$ $\hat{k}$, thus improving upon $\mathcal{E}$.

Case 2.2: $k(\mu)>\hat{k}$. In this case $V\left(\hat{k}, K_{2}, \hat{y}\right)>V^{*}$. Then some type-1 individuals of $\lambda^{j}$ with some type-2 individuals of $\lambda^{i}$ can form a nation $\sigma$ with $k(\sigma)=\hat{k}$. This proves the "if" part of the theorem.

Now let $\mathcal{E}=\left\langle\lambda^{1}, \ldots, \lambda^{m}\right\rangle$ be a partition of $\mu$ with $k\left(\lambda^{j}\right) \in B \cup C$ for all $j=1, \ldots, m$. If $k(\mu) \leq \hat{k}$, all the type-2 individuals get the maximum utility consistent with a national equilibrium with respect to $\hat{y}$. Hence there is no nation with type-2 individuals that improves upon $\mathcal{E}$.

If $k(\mu)>\hat{k}$, then a nation $\sigma$ with some positive measure of type-2 individuals improves upon $\mathcal{E}$ only if $k(\sigma)<k\left(\lambda^{j}\right)$ for all $\lambda^{j} \in \mathcal{E}$. But no type-1 individual wants to form such a nation. Since type-1 individuals cannot improve upon any partition by themselves, $\mathcal{E}$ is an equilibrium.

Proof of Theorem 2: We first show existence. By construction, $C \neq \varnothing, B \neq$ $\varnothing, C \subseteq\left[K_{1}, k(\mu)\right]$, and $B \subseteq\left[k(\mu), K_{2}\right]$. If $k(\mu) \in B \cup C$, by Theorem $1,\langle\mu\rangle$ is an international equilibrium. Otherwise, by Lemma 3 there exists a partition of $\mu$ into two nations, one in $C$ and one in $B$, which by Theorem 1 constitutes an equilibrium. To show essential uniqueness, we need the following lemma.

LEMMA 5: $\quad B \cup C$ is a nonempty set with at most two elements.

Proof of Lemma 5: First we show that $C$ has exactly one element. It follows directly by definition of $C$ if $\hat{k} \geq k(\mu)$. If $\hat{k}<k(\mu)$, assume that there exist $k_{1}$ and $k_{2}$ in $C$, with $k_{1} \leq k_{2}$ (recall that $\left.C \neq \varnothing\right)$. By the definition of $C, k_{2} \in X\left(k_{1}, \hat{y}\right)$. But since $\mu$ is a well-behaved economy it must be that $k_{1}=k_{2}$. We know that $B \neq \varnothing$, and since $\mu$ is a well-behaved economy, $B$ cannot have more than two elements. If it has only one element we are done. If it has more 
than one, by the assumption that $\mu$ is well behaved, $k(\mu)$ is one of them. But then $k(\mu) \in C$.

Let $\mu$ be a well-behaved world economy. By Lemma 5, $B \cup C$ is a nonempty set with at most two elements. If $B \cup C$ is a singleton, it is clear that the essentially unique equilibrium is $\langle\mu\rangle$. So let $B \cup C=$ $\left\{k_{1}, k_{2}\right\}$ with $k_{1} \neq k_{2}$. Let $\mathcal{E}=\left\langle\lambda^{1}, \ldots, \lambda^{m}\right\rangle$ be an international equilibrium. Define the following partition of $\mu: \mathcal{E}^{\prime}=\left\langle\sigma^{1}, \sigma^{2}\right\rangle$, where

$$
\sigma^{1}=\sum_{k\left(\lambda^{j}\right)=k_{1}} \lambda^{j} \text { and } \sigma^{2}=\sum_{k\left(\lambda^{j}\right)=k_{2}} \lambda^{j} .
$$

By Theorem $1, \mathcal{E}^{\prime}$ is an international equilibrium. $\mathcal{E}^{\prime}$ is the coarsest partition of $\mu$ relative to $\mathcal{E}$. By Lemma $3, \mathcal{E}^{\prime}$ is the unique partition of $\mu$ into two economies with capital-labor ratios $k_{1}$ and $k_{2}$ respectively. Thus the coarsest partition of $\mu$ relative to any international equilibrium is independent of the equilibrium and equal to $\mathcal{E}^{\prime}$. Therefore $\mu$ has an essentially unique equilibrium.

Proof of Theorem 3: Since both world economies have the same distribution of endowments, their common capital-labor ratio is denoted by $k(\mu)$. Consider the world economy $\mu\left[y^{*}\right]$. As in Section 4.2, define $k^{*}=\max X\left(K_{1}, y^{*}\right)$ and $B\left(y^{*}\right)=X\left(k(\mu), y^{*}\right)$. Since $\mu\left[y^{*}\right]$ is a wellbehaved and U-shaped economy, and since $y^{*} \geq \max \mathcal{A}$, we have that $K_{2} \in B\left(y^{*}\right)$. Define now

$$
C\left(y^{*}\right)= \begin{cases}\left\{K_{1}\right\} & \text { if } k^{*} \geq k(\mu) \\ \left\{k \in\left[k^{*}, k(\mu)\right]: V\left(k, K_{2}, y^{*}\right)=V\left(K_{2}, K_{2}, y^{*}\right)\right\} & \text { if } k^{*}<k(\mu) .\end{cases}
$$

In a similar way define $B(\tilde{y})$ and $C(\tilde{y})$.

Note that since $\mu\left[y^{*}\right]$ and $\mu[\tilde{y}]$ are well-behaved and U-shaped, $C\left(y^{*}\right)$ and $C(\tilde{y})$ are singletons.

By Theorem $1, \lambda^{i} \in \mathcal{E}^{*}$ only if $k\left(\lambda^{i}\right) \in B\left(y^{*}\right) \cup C\left(y^{*}\right)$, and, similarly, $\lambda^{i} \in \tilde{\mathcal{E}}$ only if $k\left(\lambda^{i}\right) \in B(\tilde{y}) \cup C(\tilde{y})$. Therefore, all type-2 individuals get the same utility level of $V\left(K_{2}, K_{2}, y^{*}\right)=V\left(K_{2}, K_{2}, \tilde{y}\right)$ at any international equilibrium of $\mu\left[y^{*}\right]$ or of $\mu[\tilde{y}]$. As a consequence,

- All type-2 individuals consume the same amount of peanuts at the two equilibria considered.

Note too that, by Lemma $5, B\left(y^{*}\right) \cup C\left(y^{*}\right)$ has at most two elements. But since one of them is $K_{2}$ and the other belongs to $C\left(y^{*}\right)$, we conclude that $B\left(y^{*}\right) \cup C\left(y^{*}\right)$ has exactly two elements: $B\left(y^{*}\right) \cup C\left(y^{*}\right)=$ $\left\{k^{*}, K_{2}\right\}$, where $k^{*} \in C\left(y^{*}\right)$. Similarly, $B(\tilde{y}) \cup C(\tilde{y})$ has exactly two elements: $B(\tilde{y}) \cup C(\tilde{y})=\left\{\tilde{k}, K_{2}\right\}$, where $\tilde{k} \in C(\tilde{y}){ }^{4}$ We claim that $k^{*}<$ $\tilde{k}$. To see this, note that by definition of $C(\tilde{y})$, either $V\left(\tilde{k}, K_{2}, \tilde{y}\right)=$

\footnotetext{
${ }^{4}$ Note that if $\tilde{y}=\max \mathcal{A}$ then $\tilde{k}=k(\mu)$.
} 
$V\left(K_{2}, K_{2}, \tilde{y}\right)$ or $\tilde{k}=K_{1}$. But since by assumption there is an equilibrium nation with a positive measure of both types of individuals, $\tilde{k}>$ $K_{1}$. Therefore, $V\left(\tilde{k}, K_{2}, \tilde{y}\right)=V\left(K_{2}, K_{2}, \tilde{y}\right)$. Since $V$ is decreasing in the policy argument, we have that $V\left(\tilde{k}, K_{2}, y^{*}\right)<V\left(K_{2}, K_{2}, \tilde{y}\right)=V\left(K_{2}, K_{2}, y^{*}\right)$. Since $\mu\left[y^{*}\right]$ is a U-shaped economy, $V\left(k, K_{2}, y^{*}\right)<V\left(K_{2}, K_{2}, y^{*}\right)$ for all $k \in\left(\tilde{k}, K_{2}\right)$. Therefore we must have $k^{*}<\tilde{k}$. Since the per capita production function $f$ is increasing and strictly concave, we have that

- Total production in the world economy $\mu\left[y^{*}\right]$ is lower than total production in the world economy $\mu[\tilde{y}]$.

Since $K_{2} \in B\left(y^{*}\right)$, by Theorems 2 and 1 , all the type- 1 individuals live in nations with the same capital-labor ratio. As a result, the definition of national equilibrium with respect to $y^{*}$ implies that

- All type-1 individuals consume the same amount of peanuts at all international equilibria of $\mu\left[y^{*}\right]$.

As for $\mu[\tilde{y}]$, an analogous reasoning shows that

- All type-1 individuals consume the same amount of peanuts at all international equilibria of $\mu[\tilde{y}]$.

We have shown that in the equilibria of both world economies, type-2 individuals consume the same amount of peanuts. Moreover, within each world economy, all type-1 individuals have the same consumption plans. Additionally, in $\mu[\tilde{y}]$ total production is larger. Consequently, all the additional production is distributed equally among the type-1 individuals (who are still poorer than type-2 individuals). This completes the proof of the theorem.

\section{References}

ALESINA, A., and E. SPOLAORE (1997) On the number and size of nations, Quarterly Journal of Economics 112, 1027-1056.

BENABOU, R. (1993) Workings of a city: Location, education, and production, Quarterly Journal of Economics 108, 619-652.

BENNETT, E., and M. WOODERS (1979) Income distribution and firm formation, Journal of Comparative Economics 3, 304-317.

Bewley, T. (1981) A critique of Tiebout's theory of local public expenditures, Econometrica 49, 713-741.

BOLTON, P., and G. ROLAND (1997) The break up of nations: A political economy analysis, Quarterly Journal of Economics 112, 1057-1090.

COLE, H., and E. PRESCOTT (1997) Valuation equilibrium with clubs, Journal of Economic Theory 74, 19-39. 
CONLEY, J. P., and M. H. WOODERS (1997) Equivalence of the core and competitive equilibrium in a Tiebout economy with crowding types, Journal of Urban Economics 41, 421-440.

CUKIERMAN, A., Z. HERCOWITZ, and D. PINES (1994) The political economy of immigration, mimeo, The Eitan Berglas School of Economics, Tel-Aviv University.

ELLICKSON, B., B. GRODAL, S. SCOTCHMER, and W. ZAME (1999) Clubs and the market, Econometrica 67, 1185-1217.

EPPLE, D., and T. ROMER (1991) Mobility and redistribution, Journal of Political Economy 99, 828-858.

GREENBERG, J., and S. WEBER (1986) Strong Tiebout equilibrium under restricted preferences domain, Journal of Economic Theory 38, 101-117.

GUESNERIE, R., and C. ODDOU (1981) Second best taxation as a game, Journal of Economic Theory 25, 67-91.

HERCOWITZ, Z., and D. PINES (1991) Migration with fiscal externalities, Journal of Public Economics 46, 163-180.

KURZ, M. (1994) Game theory and public economics; in Handbook of Game Theory. Amsterdam: Elsevier Science B.V., pp. 1154-1192.

MYERS, G. M. (1990). Optimality, free mobility and the regional authority in a federation, Journal of Economic Theory 43, 107-121.

RAZIN, A., and E. SADKA (1995) Resisting migration: Wage rigidity and income distribution, American Economic Review 85, 312-316.

TIEBOUT, M. (1956) A pure theory of local expenditures, Journal of Political Economy 64, 413-424.

WESTHOFF, F. (1977) Existence of equilibria in economies with a local public good, Journal of Economic Theory 14, 84-112.

WILDASIN, D. E. (1991) Income redistribution in a common labor market, American Economic Review 81, 757-774.

WOODERS, M. (1978) Equilibria, the core, and jurisdiction structures in economies with a local public good, Journal of Economic Theory 18, 328-348. 